\title{
Direct Real-Time Cavity-Mode Assessment and Optimization Technique for Fiber Lasers
}

\author{
Yicheng Lai, W. Zhang, J. A. R. Williams, Member, IEEE, and I. Bennion, Member, IEEE
}

\begin{abstract}
A simple technique for direct real-time assessment of a fiber laser cavity-mode condition during operation is demonstrated. Mode stabilization and optimization with this cavity-mode monitoring and conditioning feedback scheme shows significant improvements to the output performance.
\end{abstract}

Index Terms-Gratings, laser modes, optical fiber lasers, optimization methods.

\section{INTRODUCTION}

$\mathbf{L}$ INEAR cavity rare-earth-doped fiber grating lasers are considered a potential alternative to their semiconductor counterparts. Besides output performance merits like narrow optical linewidth, they also inherit advantages like engineering simplicity inherent to the fiber Bragg grating (FBG). Ideally in a single-mode fiber laser configuration, the cavity-mode structure, largely defined by the FBGs, yields a dominant resonance mode at the Bragg center to achieve high-level performance like low threshold and high side-mode suppression [1]. In reality, fiber gratings are sensitive to ambient conditions and any perturbation will detune the cavity resonance-mode structure. Furthermore, in particular for fiber lasers with phase-shifted grating structures (e.g., distributed feedback (DFB) fiber laser), refractive index variations (hence, spectral drift) associated with the out-diffusion of hydrogen during the annealing stage of the fabrication process can deviate the induced phase shift [2] thereby detune the cavity-mode conditions. Most significantly, the pump-induced thermooptic effects in rare-earth-doped fibers during operation can introduce Bragg wavelength shift and grating chirp that consequently influence the resonance-mode performance or even inhibit lasing operation. Previous studies have all highlighted the dependency of the fiber laser performance on the ambient conditions and the pump power (e.g., [3]).

In contrast to semiconductor lasers, a direct real-time cavity-mode assessment and optimization scheme for fiber grating lasers remains a missing key element for optimized operation. For applications involving wavelength tuning, mode condition monitoring, and feedback control are vital to prevent operating near the onset of mode-hopping and to ensure performance integrity over the operation band. Reported stabilization schemes (e.g., [4]) only address long-cavity-length ring laser

Manuscript received July 18, 2003; revised October 12, 2003.

The authors are with the Photonics Research Group, School of Engineering and Applied Science, Aston University, Birmingham B4 7ET, U.K. (e-mail: laiy@ aston.ac.uk).

Digital Object Identifier 10.1109/LPT.2003.823091 structures and require additional filters or phase modulators Proposed schemes using interferometric techniques or an acetylene gas cell (e.g., [5]), however, only maintain the obtained output frequency. Without determining the exact operation regime, these methods do not optimize the lasing mode and may be inadequate for robust applications.

In this letter, we propose and demonstrate a novel technique that allows direct real-time assessment of a fiber laser cavity conditions during operation. It exploits the concept and simplicity of superimposed fiber grating structures by inscribing onto the fiber laser an identical secondary nonlasing cavity. Utilizing the spectral information of the secondary structure, a truly cavity-mode conditioning feedback mechanism is realized. The proposed scheme is applicable to all linear cavity configurations and is particularly useful for short structural length designs. For illustration, optimization for single-mode operation of a highly doped $\mathrm{Er}-\mathrm{Yb}$ distributed Bragg reflector (DBR) fiber laser and a DFB fiber laser for dense wavelength-division-multiplexing applications are presented. Without any environmental conditioning or isolation, stable single-mode output with power fluctuations of $<0.05 \mathrm{~dB}$ and negligible wavelength drift is achieved despite under the strong influence of the pump-induced thermal effects within the Er-Yb DBR fiber laser. Results further illustrate that the optimized DFB fiber laser exhibits higher efficiency, lower threshold, and better relative intensity noise (RIN).

\section{BACKGROUND}

It has been demonstrated that for dual overwritten fiber grating laser structures, the spectra of the individual cavities can remain independent of each other [6]. More importantly, perturbations incurred in the laser are imposed simultaneously onto both cavities. By designing the superimposed secondary grating structure such that its lasing threshold is not reached, it will remain passive during operation and only serves to mirror the mode conditions in the active primary cavity. The high finesse resonance peaks in the passive grating structure allow continuous, accurate, and even remote wavelength and mode condition monitoring to a very high accuracy and resolution. Determination of whether the laser is near a mode-hop or detuned from the Bragg center is achieved by examining this correlated passive secondary cavity. Mode stabilization and optimization feedback based on piezos or peltiers can, hence, be implemented using the error signal generated by the deviation of the Bragg center and the resonance peak position from the desired optimum in the secondary grating structure. 


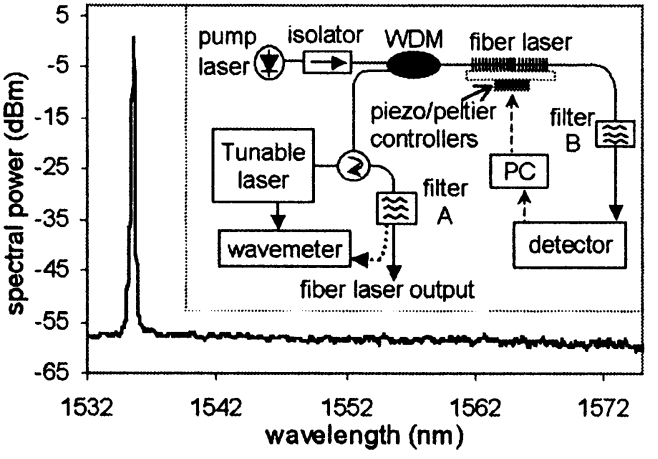

Fig. 1. Output spectrum of the DBR fiber laser. Inset shows the experimental setup for the fiber laser.

\section{EXPERIMENT}

The DFB fiber laser structure consisted of a 45-mm-long $\pi$ phase-shifted FBG (Bragg wavelength $=1545.8 \mathrm{~nm}, \kappa L \sim$ 6.1) superimposed with an identical secondary grating structure (Bragg wavelength $=1569.5 \mathrm{~nm}, \kappa L \sim 3.2$ ) at the same physical location in a Er-Yb fiber (absorption at $980 \mathrm{~nm}=230 \mathrm{~dB} / \mathrm{m}$ ). The phase shift was induced by post processing over a $0.7-\mathrm{mm}$ center section of the grating. The fiber laser was mounted on a 50-mm-long thin metal plate with temperature gradient (hence, heat dissipation rate) along the laser cavity controlled by two peltiers on each end of the plate. A copper filament wire with one end thermally bonded to a third peltier was placed over the phase-shifted region to thermally adjust the phase deviations.

On the other hand, the DBR fiber laser structure consisted of two 25-mm-long uniform FBGs (Bragg wavelength $=1535.5 \mathrm{~nm}, \kappa L \sim 2.1)$ in standard fiber and a 30-mm highly doped $\mathrm{Er}-\mathrm{Yb}$ fiber (absorption at $979 \mathrm{~nm}=730 \mathrm{~dB} / \mathrm{m}$ ) as the gain medium. At the same physical location, an identical secondary grating structure (Bragg wavelength $=1566.9 \mathrm{~nm}$, $\kappa L \sim 1.4)$ was superimposed. A short piezo $(7 \mathrm{~mm})$ was adhered to the $\mathrm{Er}-\mathrm{Yb}$ fiber to vary the cavity path length as a means to alter the cavity mode conditions. In a separate experiment, over an applied voltage of $0-6.2 \mathrm{~V}$, the piezo induced a strain of $0.004 \%$ to shift the cavity resonance-mode structure half a free spectral range (FSR). Note that the exact Bragg wavelength selection for the secondary cavity is not critical so long as the cavity does not reach lasing threshold during operation.

The operation setup of the fiber lasers is shown in Fig. 1 (inset). A scanning tunable laser ( $\max 100 \mathrm{~nm} / \mathrm{s}, 1-\mathrm{pm}$ resolution calibrated by the wavemeter) and a detector are used to probe real-time the spectral profile of the passive secondary cavity during operation. A computer acquires the spectral data from the detector and applies control signals to the peltier-piezo drivers to alter the cavity-mode conditions. Other FBG-based interrogation techniques and dedicated electronics can otherwise be adopted to simplify the setup. Optical filters A and B were used to isolate the probe signal and the fiber laser output respectively. No special attention was necessary to isolate any part of the setup from ambient perturbations.

In the experiment for the DBR fiber laser, lasing operation only occurs from the primary cavity expectedly, as shown in Fig. 1. The spectral profiles of the primary and secondary cavities prior to operation are shown in Fig. 2(a) and the inset
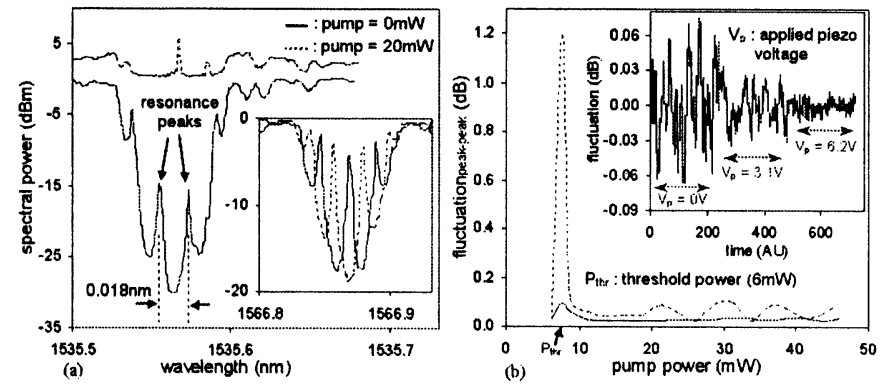

Fig. 2. (a) Spectral profiles of the primary and secondary (inset) DBR fiber laser cavities at different pump powers. (b) Power fluctuations with and without mode optimization feedback.

(solid traces), respectively. Each resonance peak in the primary laser cavity corresponds to a potential lasing longitudinal mode. Within the stopband of the primary laser cavity, there are two dominant resonance modes with an FSR of $18 \mathrm{pm}$, as indicated in Fig. 2(a). Without feedback control, as the pump power increased from 0 to $50 \mathrm{~mW}$, the resonance peaks shift continuously within the Bragg grating spectrum due to the pump-induced thermooptic effects in the Er-Yb fiber measured $\sim 0.87 \times 10^{-5} \mathrm{~mW}$ in both cavities. As shown in Fig. 2(a) (dotted trace), direct spectral characterization of the active primary cavity during operation is not feasible as the lasing mode saturates the detector and obscures the spectral features around it. Resonance-mode structure in the passive secondary cavity depicted in the inset of Fig. 2(a) (dotted trace), however, remains clearly resolved. Due to the detuning of the dominant lasing mode, the fiber laser exhibits transitions from single-mode to multimode operation exemplified by occurrences of an intermodal beat signal at $2.33 \mathrm{GHz}$. As shown in Fig. 2(b) (dotted trace), output power instability associated with mode competition and mode-hopping were encountered together with wavelength fluctuations of $>18 \mathrm{pm}$ over the entire pump power range. A simple feedback control based on detecting the deviation of the resonance peaks from its optimal positions in the secondary cavity was implemented. The optimum secondary cavity resonance-mode structure was such that the dominant resonance mode in the primary cavity residues at the Bragg center [1]. As illustrated in Fig. 2(b) (inset), output power fluctuation in the multimode operation regime is suppressed as the piezo, with increasing applied voltage, alters the cavity path length to restore the desired cavity-mode condition. Over the entire pump power range, stable single-mode operation is obtained with power fluctuations $<0.05 \mathrm{~dB}$ (Fig. 2(b), solid trace) and wavelength deviation $<1 \mathrm{pm}$ (limited by the measurement setup). Furthermore, output power fluctuation near threshold is also alleviated with optimization and shows an order of magnitude improvement in stability.

Prior to the experiment for the DFB fiber laser, no lasing operation is obtained with the laser suspended in air. The measured spectral profile of the primary laser cavity (Fig. 3(a), Trace i) compared to its initial profile in the inset of Fig. 3(a) highlight a detuned phase shift (i.e., resonance peak) as a result of the annealing process. More importantly, pump-induced thermal effects in the $\mathrm{Er}-\mathrm{Yb}$ fiber further distort the grating structure as 

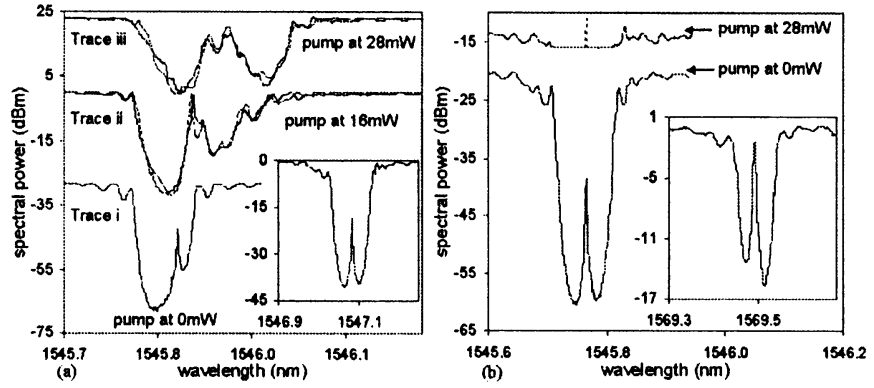

Fig. 3. (a) Measured and simulated (dotted) profiles of the DFB fiber laser primary cavity under the influence of pump-induced thermal effects. The spectra are vertically offset for clarity. Inset shows the spectral profile before annealing process. (b) Spectral profiles of the primary and secondary (inset) cavities at different pump powers with the optimization feedback.

exemplified by the measured and simulated spectral profiles of the primary cavity at 16 and $28 \mathrm{~mW}$ in Fig. 3(a) Trace ii and iii, respectively. Results indicate an exponential temperature distribution along the cavity with a peak temperature difference of $14{ }^{\circ} \mathrm{C}$ at $28 \mathrm{~mW}$.

With the thermal conditioning feedback scheme in operation, the optimized spectral profiles of the primary and the secondary cavities are shown in Fig. 3(b) and the inset, respectively. The control mechanism detects the expansion in the grating stopband (i.e., chirping) and applies a cooling gradient along the cavity by the two peltiers on each end of the plate. The thin filament wire thermally optimizes the deviation in the phase shift manifested by the variation of the resonance peak from the Bragg center. Note that due to the fact that the two cavities centered at different Bragg wavelengths and that the induced phase shift is a function of the wavelength, the transmission peak position, hence, differs for the two cavities for the same amount of induced index. Nevertheless, the feedback control maintains the secondary cavity spectral profile, thereby stabilizing and optimizing the resonance mode in the primary cavity during operation. Similar to the DBR fiber laser, direct spectral characterization of the active primary cavity in the DFB fiber laser during operation is not feasible, as shown in Fig. 3(b) (dotted trace). The spectral profile in the passive secondary cavity, however, remains highly resolved. The fiber laser delivers 1-mW output at 52-mW pump power with a slope efficiency of $\sim 3 \%$ (Fig. 4 , Trace i) limited by the fiber properties. Output wavelength deviation measured over the entire pump power range was $<1 \mathrm{pm}$ (limited by the measurement setup). In the absence of optimization and with only uncontrolled thermal dissipation through the metal plate, the fiber laser delivers only $0.37 \mathrm{~mW}$ at $52-\mathrm{mW}$ pump power with a slope efficiency of $1.2 \%$ (Fig. 4, Trace ii). Furthermore, the threshold increases by $4 \mathrm{~mW}$ and a wavelength drift of $27 \mathrm{pm}$ over the entire pump power range is measured.

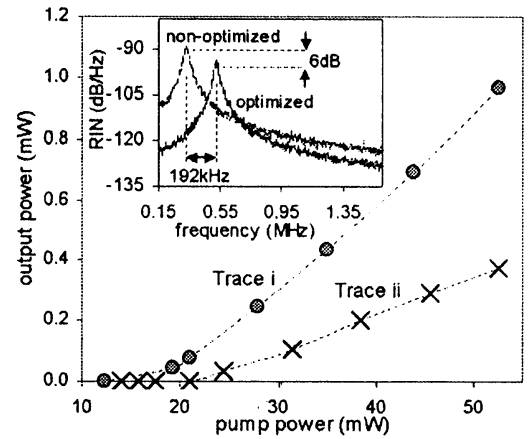

Fig. 4. Output efficiency curves and RIN (inset) measurement of the DFB fiber laser with and without mode optimization feedback.

Spectral information from the secondary cavity indicates both the localized heat-induced index change at the phase shift region of $\sim 5 \times 10^{-5}$ and an overall Bragg wavelength shift of $25 \mathrm{pm}$ account for the wavelength drift. Interestingly, comparing the RIN measurement (inset of Fig. 4) at 40-mW pump power, it is evident that the optimized fiber laser exhibits 6-dB improvement in noise performance accompanied by an increase in the relaxation oscillation frequency of $192 \mathrm{kHz}$. The variations in the RIN spectrum can be explained by the enhancement of the cavity photon lifetime under the optimized condition [7].

\section{CONCLUSION}

A simple and effective technique to assess real-time a fiber grating laser cavity condition during operation has been demonstrated. Results highlight the importance of cavity-mode conditioning feedback for optimized output performance.

\section{REFERENCES}

[1] G. A. Ball and W. H. Glenn, "Design of a single-mode linear cavity erbium fiber laser utilizing bragg reflectors," J. Lightwave Technol., vol. 10, pp. 1338-1343, Oct. 1992.

[2] B. Malo, J. Albert, K. O. Hill, F. Bilodeau, and D. C. Johnson, "Effective index drift from molecular hydrogen diffusion in hydrogen-loaded optical fibers and its effect on Bragg grating fabrication," Electron. Lett., vol. 30, no. 5, pp. 442-444, 1994.

[3] O. Hadeler, E. Rønnekleiv, M. Berendt, and M. Zervas, "Temperature distribution along DFB fiber lasers," IEE Opt. Fiber Grating Colloquium, pp. 15/1-15/6, 1999.

[4] H. Sabert, "Active stabilization of singlemode operation in a fiber laser," Electron. Lett., vol. 29, no. 11, pp. 1004-1005, 1993.

[5] S. L. Gilbert, "Frequency stabilization of a tunable erbium-doped fiber laser,” Opt. Lett., vol. 16, no. 3, pp. 150-152, 1991.

[6] Y. Lai, W. Zhang, J. A. R. Williams, and I. Bennion, "An optical millimeter wave fiber laser," presented at the Opt. Fiber Commun., Atlanta, GA, 2003, Paper TuL5.

[7] M. Ding and P. K. Cheo, "Analysis of Er-doped fiber laser stability by suppressing relaxation oscillation," IEEE Photon. Techol. Lett., vol. 8, pp. 1151-1153, Sept. 1996. 